ISAHP 1996, Vancouver, Canada, July 12 - 15, 1996

\title{
DECISIONS WITH THE ANALYTIC NETWORK PROCESS (ANP)
}

\author{
Thomas L. Saaty \\ University of Pittsburgh \\ SAATY@vms.cis.pitt.edu
}

\begin{abstract}
The Analytic Hierarchy Process (AHP) is a theory for decision making involving ratio scale measurement in hierarchic structures. The AHP elicits judgments from people or uses actual ratio scale measurements either directly or by interpreting existing measurements. Judgments are made on paired comparisons of elements on a common property or criterion. For accuracy, the elements compared must be homogeneous (relatively close) and if far apart then they are grouped into clusters with a pivot element from one cluster to the next.
\end{abstract}

The Analytic Network Process (ANP) is a new theory that extends the AHP to cases of dependence and feedback and generalizes on the supermatrix approach introduced in my 1980 book on the Analytic Hierarchy Process. It allows interaction and feedback within clusters (inner dependence) and between clusters (outer dependence). Feedback can better capture the complex effects of interplay in human society. The ANP provides a thorough framework to include clusters of elements connected in any desired way to investigate the process of deriving ratio scale priorities from the distribution of influence among elements and among clusters. The AHP becomes a special case of the ANP. Although many decision problems are best studied through the ANP, it is not true that forcing an ANP model always yields better results than using the hierarchies of the AHP. There are examples to justify the use of both. We have yet to learn when the shortcut of the hierarchy is justified, not simply, on grounds of expediency and efficiency, but also for reasons of validity of the outcome.

The ANP is implemented in the software ECNET and has been applied to yarious problems both to deal with decisions and to illustrate the uses of the new theory. The ANP is a coupling of two parts. The first consists of a control hierarchy or.network of criteria and subcriteria that control the interactions in the system under study. The second is a network of influences among the elements and clusters. The network varies from criterion to criterion and a supermatrix of limiting influence is computed for each control criterion. Finally, each of these supermatrices is weighted by the priority of its control criterion and the results are synthesized through addition for all the control criteria.

In addition, a problem is often studied through a control hierarchy or system of benefits; a second for costs, a third for opportunities, and a fourth for risks. The synthesized results of the four control systems are combined by taking the quotient of the benefits times the opportunities to the costs times. the risks to determine the best outcome. Here is a rough outline of the steps of the ANP. followed by two lists of concepts of both the AHP and the ANP. 


\section{Outline of ANP Steps}

1. Determine the control hierarchies including their criteria for comparing the components of the system and their subcriteria for comparing the elements of the system. One hierarchy for benefits, a second for costs, a third for opportunities, and a fourth for risks. If in some cases, a hierarchy does not apply because its criteria are all unimportant, leave out that hierarchy. For benefits and opportunities, ask what gives the most benefits or presents the greatest opportunity to influence fulfillment of that control criterion. For costs and risks, ask what incurs the most cost or faces the greatest risk. Sometimes, the comparisons are made simply in terms of benefits, opportunities, costs, and risks in the aggregate without using criteria and subcriteria.

2. For each control criterion or subcriterion, determine the clusters of the system with their elements.

3. To better organize the development of the model, as well as you can and roughly, for each control criterion, number and arrange the clusters and their elements in a convenient way (perhaps in a column). Use the identical label to represent the same cluster and the same elements for all the control criteria.

4. Determine the approach you want to follow in the analysis of each cluster or element, being influenced by other clusters and elements, or influencing other clusters and elements with respect to a criterion. The sense (being influenced or influencing) must apply to all the criteria for the four control hierarchies.

5. For each control criterion, construct a three column table placing each cluster label in the middle column. List in the left column on a line all the clusters that influence the cluster, and in the column on the right those clusters which it influences.

6. Following each entry in the table above, perform paired comparisons on the clusters as they influence each clister and on those that it influences, with respect to that criferion. The derived weights are used later to weight the elements of the corresponding column clusters of the supermatrix' corresponding to the control 'criterion. Assign a-zero when there is no influence.

7. Perform paired comparisons on the'elements within the clusters themselves according to their influence on each element in another cluster they are connected to (or elements in their own cluster). The comparisons are made with respect to a criterion or subcriterion of the control hierarchy.

8. For each control criterion, construct the supermatrix by laying out the clusters in the order they are numbered and all the elements in each cluster both vertically on the left and horizontally at the top. 'Enter in the appropriate position the priorities derived from the paired comparisons as parts (subcolumns) of the corresponding column of the supermatrix.

9. Compute the:limiting priorities of each supermatrix according to whether it is irreducible (primitive or imprimitive [cyclic]) or it is reducible with one being a simple or a multiple root and whether the system is cyclic or not.

10. Synthesize the limiting priorities by weighting each limiting supermatrix by the weight of its control criterion and adding the resulting supermatrices.

11. Repeat the synthesis for each of the four control hierarchies: one for benefits, one for costs, a third for opportunities, and a fourth for risks.

12. Synthesize the results from the four control hierarchies by multiplying the benefits by the opportunities and dividing by the costs multiplied by the risks. hen, réad off the highest priority alternative or the desired mix of alternatives. 


\section{Concepts of the Analytic Hierarchy Process}

1. Details of the problem, goal, subgoals, time horizons, scenarios, actors and stakeholders, their objectives and policies, criteria, subcriteria, attributes, and alternatives.

2. Hierarchic structure.

3. Judgments - absolute numbers, homogeneity, clustering, pivot elements, tangibles and intangibles.

4. Comparisons, dominance and reciprocity with respect to an attribute, inconsistency and the eigenvector, use of actual measurements.

5. The number of judgments; how to take fewer judgments.

6. Derived ratio scales - in AHP the priorities are derived and are proven to belong to a ratio scale.

7. Interval judgments, stochastic judgments.

8. Synthesis - multilinear forms - density.

9. Rank - The dominance mode, the performance mode with respect to an ideal.

10. Absolute measurement - rating alternatives one at a time.

11. Benefits, opportunities, costs and risks hierarchies.

12. Parailel with human thinking - neural firing creates awareness and intensityof stimuli for both tangibles and intangibles. Measurements are data to be interpreted.

13. Group Decision Making and the reciprocal property; Pareto optimality: if each prefers A to B, then the group does.

14. Sensitivity Analysis.

15. Learning and revision as a process.

\section{Concepts of the Analytic Network Process}

1. Details of the Problem - Feedback, inner and outer dependence.

2. Influence with respect to a criterion.

3. The control hierarchy or system.

4. The supermatrix.

5. The limiting supermatrix and limiting priorities.

6. Primitivity, irreducibility, cyclicity.

7. Stochasticity, comparing the clusters.

8. Synthesis for the criteria of a control hierarchy or a control system.

9. Synthesis for benefits, costs, opportunities, and risks control hierarchies.

10. Formulation to compute the limit.

11. Relation to Neural Network Firing - the continuous case.

12. The density of neural firing and distributions and their application to reproduce visual images and symphonic compositions. Further research in this area is needed. 